\title{
IMPACTO DO EXERCíCIO FÍSICO COM AUXILIO DE EXERGAME SOBRE O ESTADO NUTRICIONAL E A PRESSÃO ARTERIAL DE ADOLESCENTES COM EXCESSO DE PESO
}

\author{
IMPACT OF PHYSICAL EXERCISE WITH THE EXERGAME \\ ASSISTANCE ON THE NUTRITIONAL STATE AND BLOOD \\ PRESSURE OF OVERWEIGHT ADOLESCENTS
}

\author{
Geisielly Raquel da Cruz Aguiar ${ }^{1}$ \\ Fernanda Caroline Tavares de Melo $^{2}$ \\ Morgana Monteiro Pimentel ${ }^{3}$ \\ Gabrielle Sousa Marques ${ }^{4}$ \\ Carla Campos Muniz Medeiros ${ }^{5}$ \\ Danielle Franklin de Carvalho ${ }^{6}$
}

RESUMO: OBJETIVO: Avaliar o impacto do exercício físico, realizado com auxílio de exergame, sobre o estado nutricional e a pressão arterial de adolescentes com excesso de peso. MÉTODOS: Estudo de intervenção quase-experimental, realizado com 51 adolescentes de 15 a 19 anos, portadores de sobrepeso ou obesidade, matriculados em escolas públicas de ensino médio do município de Campina Grande, Paraíba. A intervenção consistiu em participar de jogos de dança durante 50 minutos, três vezes por semana, ao longo de oito semanas. Utilizou-se a plataforma XBOX 360, com acessório Kinect (Microsoft ${ }^{\circledR}$ ) e o Just Dance foi o jogo selecionado. A pressão arterial (PA) foi aferida antes e depois da intervenção, e comparada através do teste de McNemar, no SPSS 22.0. Foi adotado o Intervalo de Confiança de 95\%. RESULTADOS: Após a intervenção, um adolescente mudou o estado nutricional de obesidade para sobrepeso e três tornaram-se eutróficos. Não se verificou alteração estatisticamente significante na pressão arterial. CONCLUSÃO: A intervenção com uso de exergame não causou impacto na pressão

\footnotetext{
${ }^{1}$ Graduada em Enfermagem. Universidade Estadual da Paraíba, Campina Grande, Paraíba, Brasil.

${ }^{2}$ Graduada em Enfermagem. Universidade Estadual da Paraíba, Campina Grande, Paraíba, Brasil.

${ }^{3}$ Graduada em Enfermagem. Universidade Estadual da Paraíba, Campina Grande, Paraíba, Brasil.

${ }^{4}$ Graduada em Enfermagem. Universidade Estadual da Paraíba, Campina Grande, Paraíba, Brasil.

${ }^{5}$ Doutora em Saúde da Criança e do Adolescente. Universidade Estadual da Paraíba, Campina Grande, Paraíba, Brasil.

${ }^{6}$ Doutora em Saúde da Criança e do Adolescente. Universidade Estadual da Paraíba, Campina Grande, Paraíba, Brasil.
} 
arterial dos adolescentes, porém, observou-se uma mudança no estado nutricional de quatro adolescentes. Acredita-se que a prática por um período maior poderia implicar em melhores resultados para a saúde.

Palavras chave: Adolescente; Exercício Físico; Obesidade; Pressão Arterial.

ABSTRACT: OBJECTIVE: Evaluate the impact of physical exercise, performed with exergame, on the nutritional status and blood pressure of overweight adolescents. METHODS: A quasi- experimental study was carried out with 51 adolescents aged 15 to 19 years, who were overweight or obesity, enrolled in public high schools in the city of Campina Grande, Paraíba. The intervention consisted of participating in games of dance for 50 minutes, three times a week, over eight weeks. We used the

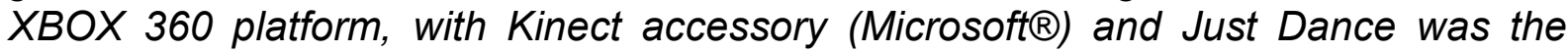
game selected. Blood pressure $(B P)$ was measured before and after the intervention, and compared using the McNemar test in SPSS 22.0. The Confidence Interval of 95\% was adopted. RESULTS: After the intervention, one adolescent changed the nutritional status from obesity to overweight and three became eutrophic. There was no statistically significant change in blood pressure. CONCLUSION: The intervention with exergame did not have an impact on the blood pressure of adolescents, however, a change in the nutritional status of four adolescents was observed. It is believed that the practice over a longer period could imply better health outcomes.

Keywords: Adolescent; Exercise; Obesity; Arterial Pressure. 


\section{INTRODUÇÃO}

De acordo com a Organização Mundial da Saúde (OMS) o mundo apresenta uma população de 1,2 bilhões de adolescentes com idades entre 10 e 19 anos, além disso, esse período é considerado como uma fase crítica da vida e um dos períodos que apresentam mais desafios para esses indivíduos, tendo em vista que durante essa fase os jovens tornam-se independentes, são desenvolvidas habilidades sociais, além do aprendizado de comportamentos que irão perdurar por toda vida (WHO, 2018).

Nesta fase tem sido comum o aparecimento de diversas doenças crônicas não transmissíveis (DCNT), frequentemente associadas à prática reduzida de atividade física (AF), à adoção de hábitos sedentários e ao consumo de alimentos com alta densidade energética. Dentre elas, a obesidade tem se transformado em uma epidemia mundial, representando um importante fator de risco cardiovascular e contribuindo para o aumento de diversas morbidades associadas, como a Hipertensão Arterial Sistêmica (HA), (FRIEDEMANN et al., 2012).

Segundo dados da OMS, em 2016 mais de 340 milhões de crianças e adolescentes com a faixa etária de 5 a 19 anos estavam com excesso de peso ou obesas (WHO, 2018). Ademais, foi verificado a partir dos resultados do Estudo de Riscos Cardiovasculares em Adolescentes (ERICA), no qual foram envolvidos diversos municípios do território brasileiro, que $25,0 \%$ dos adolescentes brasileiros matriculados em escolas de municípios com mais de 100 mil habitantes estavam com excesso de peso e $24 \%$ apresentavam pressão arterial elevada (préhipertensão ou hipertensão) (BLOCH et al., 2016).

A presença dessas doenças em fases precoces do ciclo vital implica na elevação do risco de desenvolvimento de doenças crônicas não transmissíveis (DCNT) na vida adulta (BIRO; WIEN, 2010; CRAIGIE et al., 2011). Ademais, a identificação e prevenção dessas doenças, ainda no período da infância, são de 
extrema valia para que sejam evitados possíveis agravos que podem ocorrer no futuro (MORAES et al., 2014).

A literatura científica mostra que a prática de atividade física na infância pode estar associada a um risco reduzido de desenvolver sobrepeso e obesidade, sendo uma estratégia de saúde de extrema importância, pois, além do bem-estar, possui efeitos positivos sobre a saúde cardiovascular e musculoesquelética (CABRERA, 2014). Dessa forma, para a melhoria da aptidão cardiorrespiratória, muscular e da saúde óssea, recomenda-se, pelo menos, 150 minutos de atividade física semanal, considerado seu lazer, trabalho e deslocamento (QUEIROGA, 2016). Além disso, de acordo com Marques et al (2015), o exercício físico exerce grande capacidade de influência favorável sobre o perfil tensional.

Diante do cenário de frequente diminuição da prática de $\mathrm{AF}$, o uso do exergame apresenta-se como uma ferramenta tecnológica que proporciona essa prática. O exergame ou vídeo game ativo (VGA) é definido como sendo uma tecnologia que utiliza jogos interativos para aumentar o desempenho do exercício, no qual exige que os jogadores relacionem-se fisicamente com avatares na tela por meio da realização de variados movimentos corporais, como saltar, chutar e esquivar-se, tornando os jogadores fisicamente ativos, além de corroborar para o aumento dos níveis de atividade física dos jogadores e afetar beneficamente sua saúde em geral (GAO; CHEN; STODDEN, 2015).

Não obstante, em estudos realizados com os VGAs, percebeu-se que eles podem ser empregados como ferramenta terapêutica, proporcionando uma distração para os indivíduos que os utilizam influenciando na diminuição dos níveis de estresse e quando utilizado como forma de atuação fisioterápica, seu uso pode auxiliar no melhoramento do equilíbrio, postura e atividade funcionais diárias, corroborando para manutenção ou melhoramento da saúde. (NILSSON et al., 2013; MAILLOT; PERROT; HARTLEY, 2012). Além disso, desenvolve efeitos positivos nos aspectos psicológicos das pessoas, abrangendo autoeficácia, imagem corporal, capacidade escolar e social. (NILSSON et al., 2013; MAILLOT; PERROT; HARTLEY, 2012).

Tendo em vista que a diminuição da prática de atividade física contribui para o crescente aumento da obesidade e do sobrepeso, com repercussão sobre diversos 
parâmetros cardiometabólicos, dentre eles o aumento da pressão arterial, dessa forma, é de fundamental importância à realização de estudos que avaliem esses parâmetros tendo em vista que o aumento obesidade e pressão arterial na infância podem acometer diversos agravos na vida adulta, sendo necessária a utilização de ferramentas preventivas e de auxilio no tratamento, dentre elas a prática de atividade física. Logo, o presente estudo objetivou avaliar o impacto do exercício físico realizado com o auxílio de exergame sobre o estado nutricional e a pressão arterial de adolescentes escolares com sobrepeso ou obesidade de Campina Grande-PB.

\section{MÉTODOS}

Estudo de intervenção quase-experimental, realizado com 51 adolescentes com sobrepeso ou obesidade de escolas públicas de ensino médio do município de Campina Grande, Paraíba, na faixa etária de 15 a 19 anos.

A triagem (para identificação dos casos de excesso de peso) foi realizada entre junho e julho de 2016, em escolas públicas de grande porte (igual ou superior a 500 alunos) (TENÓRIO et al., 2010), com turmas diurnas, da zona urbana do município de Campina Grande-PB. Já a intervenção realizou-se entre os meses de setembro e novembro de 2016. As escolas foram selecionadas por conveniência, de acordo com as maiores prevalências de excesso de peso, verificada em estudo anterior (RAMOS, 2015).

Adotou-se como critérios de inclusão o adolescente estar regularmente matriculado e frequentando as escolas selecionadas e cursando o primeiro ou o segundo ano do ensino médio; estar dentro da faixa etária determinada; e apresentar excesso de peso corporal.

Como critérios de exclusão foram considerados: ter alguma limitação motora ou mental que impedisse a participação nas atividades da intervenção; possuir alguma alteração metabólica severa que exigisse o uso de medicamentos ou tratamento específico que alterasse o metabolismo lipídico ou glicídico; possuir asma 
com crise recente (dois meses anteriores à coleta de dados) e/ou relato de broncoespasmo induzido pelo exercício; ser usuário de videogame ativo; e estar gestante. No caso de desenvolvimento de algum dos critérios de exclusão ao longo do estudo, isso implicaria no desligamento do adolescente da pesquisa.

A intervenção foi realizada através da prática de exercícios com auxílio de um videogame ativo e foi supervisionada e monitorizada. A intensidade foi moderada, frequência semanal de três vezes (nas escolas) e duração de 50 minutos cada sessão, por um período de oito semanas. Os jogos foram disponibilizados em salas destinadas para tal finalidade nas escolas selecionadas, em horários nos turnos da manhã e da tarde.

Foi utilizada a plataforma XBOX 360, com acessório Kinect $\left(\right.$ Microsoft $^{\circledR}$ ) e o Just Dance foi o jogo selecionado, pois além de atender ao critério de intensidade moderada para o exercício, é relatado na literatura como o que desperta maior interesse entre os adolescentes (PEREIRA et al., 2012; BIDDISS; IRWIN, 2010). O estado nutricional foi avaliado através do índice de massa corporal (IMC), construído a partir da razão do peso (em $\mathrm{Kg}$ ) pelo quadrado da altura (em metros).

A categorização do estado nutricional baseou-se no escore-z de IMC-Idade para adolescentes de 10 a 18 anos, foi preconizada a classificação da Organização Mundial da Saúde (OMS), sendo sobrepeso os adolescentes com escore $Z \geq+1$ e $\leq$ +2 e obesidade os valores correspondentes ao escore $Z>+2$ e $\leq+3$. Para os maiores de 18 anos, foi utilizado o IMC, convenciona-se chamar de sobrepeso o IMC de 25 a 29,9 kg/m² e obesidade o IMC maior ou igual a $30 \mathrm{~kg} / \mathrm{m}^{2}$ e de excesso de peso o IMC maior ou igual a $25 \mathrm{~kg} / \mathrm{m}^{2}$ (incluindo a obesidade) (ABESO, 2016). O peso foi determinado através de balança digital $\left(\right.$ Tonelli $\left.^{\circledR}\right)$, com precisão de $100 \mathrm{~g}$; e a altura através de antropômetro fixo $\left(\operatorname{Seca}^{\circledR}\right)$, com acuidade de um centímetro.

A pressão arterial (PA) foi verificada com tensiômetro semiautomático da marca OMRON ${ }^{\circledR}$ e caracterizada como elevada quando os valores da PA sistólica e/ou diastólica foram iguais ou superiores ao percentil 90, para idade, sexo e percentil de estatura, com base nas tabelas específicas para crianças e adolescentes. Além disso, os valores de PA sistólica e diastólica iguais ou acima de $130 \mathrm{mmHg}$ ou $85 \mathrm{mmHg}$, respectivamente, foram considerados elevados, independente do percentil 90 (SBC, 2010). 
Inicialmente, realizou-se uma análise descritiva para caracterização da população estudada através de frequência relativa e absoluta. Em seguida, realizouse o teste de Kolmogorov-Smirnov, a fim de avaliar o tipo de distribuição das variáveis. Posteriormente, foi realizado o teste de McNemar, com o intuito de avaliar o impacto da intervenção sobre o estado nutricional e a PA; as variáveis também foram avaliadas de forma contínua, através do teste $t$ pareado. As análises estatísticas foram realizadas no SPSS 22.0, adotando-se o intervalo de confiança de $95 \%$.

A pesquisa foi desenvolvida de acordo com a Resolução 466/2012 do Conselho Nacional de Saúde, aprovada pelo Comitê de Ética em Pesquisa da Universidade Estadual da Paraíba (CAAE: 56118616.1.0000.5187) em 30/05/2016. Foram explicados os procedimentos de pesquisa aos adolescentes e entregue uma carta de esclarecimentos e os termos de consentimento livre e esclarecido (TCLE) e de assentimento (TA).

\section{RESULTADOS}

A amostra foi composta por 51 adolescentes, sendo a maioria do sexo feminino (60,0\%); pertencente às classes econômicas C e D (60,0\%) e com sobrepeso (74,5\%). Após a intervenção, um adolescente mudou o estado nutricional de obesidade para sobrepeso e três tornaram-se eutróficos.

Com relação à pressão arterial, no início do estudo, a pressão arterial sistólica (PAS) e diastólica (PAD) estavam alteradas em 5,6\% $(n=3)$ e 3,7\% ( $n=2)$ dos adolescentes, respectivamente. Após a intervenção, as frequências de PAS e PAD alteradas passaram a ser 10,9\% $(n=6)$ e 1,8 $(n=1)$, respectivamente. Entretanto, a variação não foi estatisticamente significante nos dois casos.

As variáveis também foram avaliadas através da tendência central e sua descrição encontra-se na tabela 1. 
Tabela 1 - Variação do índice de massa corporal e da pressão arterial dos adolescentes da amostra entre o início e final do estudo. Campina Grande-PB, 2016.

\begin{tabular}{cccccc}
\hline VARIÁVEL & $\begin{array}{c}\text { BASAL } \\
\text { Média } \pm \text { DP }\end{array}$ & $\begin{array}{c}\text { MINAL } \\
\text { Média } \pm \text { DP }\end{array}$ & Média Par \pm DP & $\boldsymbol{p}$ & IC95\% \\
\hline IMC $\left(\mathrm{Kg} / \mathrm{m}^{2}\right)$ & $27,4 \pm 3,5$ & $27,5 \pm 3,8$ & $+0,030 \pm 0,940$ & 0,808 & $-0,285-0,223$ \\
PAS $(\mathrm{mmHg})$ & $113,0 \pm 12,3$ & $113,6 \pm 11,5$ & $+0,520 \pm 10,631$ & 0,729 & $-3,509-2,470$ \\
PAD $(\mathrm{mmHg})$ & $69,0 \pm 8,0$ & $69,2 \pm 9,0$ & $-0,082 \pm 9,268$ & 0,946 & $-2,518-2,695$ \\
\hline
\end{tabular}

PAS: Pressão Arterial Sistólica. PAD: Pressão Arterial Diastólica. DP: desvio-padrão. Média ${ }_{\text {Par: }}$ variação da média entre o ponto final e inicial. p: significância estatística de 5\%. IC95\%: intervalo de confiança de 95\%.

\section{DISCUSSÃO}

Após a intervenção, um adolescente mudou o estado nutricional de obesidade para sobrepeso e três tornaram-se eutróficos, fato que pode ter ocorrido devido ao dispêndio energético acarretado pelo videogame ativo. Entretanto, um estudo realizado por Perrier-Melo et al. (2016), com jovens na faixa etária entre 18 e 25 anos, no qual foi realizado uma intervenção com o VGA por um período de 6 semanas, observou que os jovens não apresentaram mudanças significativas na composição corporal, apresentando um resultado antagônico ao obtido no presente estudo.

Não obstante, em um estudo realizado por Staino, Abraham e Calvert (2013), com 54 adolescentes obesos e com sobrepeso na faixa etária entre 15 e 19 anos, no qual foi realizada uma intervenção de 20 semanas com o objetivo de avaliar se o exergame pode produzir perda de peso e melhorar os resultados psicossociais, foi possível concluir que o uso do vídeo game ativo pelos jovens com o auxilio de um parceiro aumentou significativamente o gasto calórico desses adolescentes além do aumento da perda de peso. 
Por conseguinte, averiguou-se a ausência de alteração da pressão arterial dos adolescentes após o uso do exergame como instrumento de realização de atividade física, o que pode estar atrelado ao tempo da intervenção, que pode não ter sido suficiente para provocar alterações fisiológicas que acarretassem mudança dos níveis da PA, além de fatores internos como estresse e ansiedade. Ao mesmo tempo, ressalta-se que praticamente não foram identificados casos de PA alterada na amostra. Dessa forma, é positivo que o exercício só tenha produzido alterações fisiológicas.

Em um estudo piloto realizado por Melo et al (2014) com oito adultos jovens do sexo masculino no qual foi utilizado o videogame ativo, realizado por meio de duas etapas: a primeira com a realização das medidas antropométricas e a segunda com a realização de uma sessão múltipla com quatro VGAs, observou-se uma alteração significativa na PA após a realização da atividade física com o jogo na modalidade dança e vôlei do exergame, porém, em outras modalidades de jogo, não houve mudanças significativas, já nas modalidades em geral analisou-se mudanças significativas na FC dos jovens. Resultado que se mostra divergênte ao resultado encontrado no presente estudo.

Corroborando com os nossos achados, Rauber et al (2013) desenvolveu um estudo em Brasília com oito crianças com faixa etária entre 9 e 10 anos ambos os sexos, utilizando o jogo Dance Dance Revolution (DDR), porém não foi observada redução significativa da PA. Não obstante, segundo Rauber, os valores da PAS posteriormente a uma única sessão de nível básico do videogame ativo DDR, mesmo não tendo sido significativamente inferiores aos de repouso, quando conservados ao longo do tempo podem corroborar para a prevenção do aparecimento da hipertensão arterial em crianças.

Logo, o uso do exergame como instrumento de prática de atividade física torna-se uma ferramenta de auxilio eficaz, pois de acordo com estudo realizado por Silva et al (2013) com adolescentes de 11 á 13 anos, notou-se que a chance de desenvolver HA foi quase duas vezes maior, tanto nos adolescentes considerados inativos quanto nos insuficientemente ativos. De tal modo, ao considerar a totalidade de adolescentes com baixos níveis de atividade física (inativos e insuficientemente ativos), a chance de desenvolver HA aumentará quase quatro vezes em relação aos 
adolescentes ativos. Com isso, observa-se que quanto menor o nível de atividade física maior a chance de desenvolver HA.

Pode-se constatar que o uso constante do videogame ativo pode contribuir para prevenção de futuras doenças acarretadas pelo sedentarismo, tendo em vista, que essa modalidade de atividade física torna o indivíduo ativo, o que acarreta melhorias em diversas áreas. Os exergames proporcionam variados benefícios, dentre os quais: melhoria no estilo de vida saudável, avanço nos resultados fisiológicos, desenvolvimento motor, benefícios físicos e habilidades num ambiente seguro (ROWLAND et al., 2015).

Em outro estudo realizado por Rauber et al (2014), com dezesseis crianças de 9 a 10 anos (8 meninos e 8 meninas), com o objetivo de verificar se a reatividade da pressão arterial poderia ser reduzida através de uma sessão única anterior de jogo ativo, quando comparada ao lazer sedentário, não se observou alterações significativas na PA com o uso do videogame ativo. Em contrapartida, verificou-se uma hipotensão pós-exercício quando as crianças utilizaram jogos tradicionais como "correr e pegar"; "dodge ball" e "capturar o bandeira ".

Outro fator que deve ser levado em consideração é o nível de conhecimento e prática que os adolescentes tinham com o jogo, pois segundo estudo realizado por Sell, Lillie e Taylor (2008), com 19 adolescentes universitários onde se analisou o quanto a experiência com o jogo exercia influência nas respostas fisiológicas, logo, observou-se que os participantes que tinham uma maior experiência de jogo podiam trabalhar com intensidades mais elevadas promovendo maiores valores de gastos energéticos, uma maior razão de troca respiratória e maior valor de FC, em relação aos que não tinham experiência alguma.

\section{CONCLUSÃO}

Através dos resultados obtidos pôde-se concluir que o exergame, embora não tenha modificado a média do IMC na amostra, foi capaz de mudar o estado nutricional de quatro adolescentes, que mudaram de obesidade para sobrepeso ou 
deste para a eutrofia. Ademais, embora não tenham sido registradas mudanças significativas da PA, está se tratando de uma população saudável, com poucos casos de PA alterada, de forma que mudanças fisiológicas normais são desejáveis. Acredita-se que um tempo de intervenção maior fosse necessário para acarretar os impactos positivos no IMC e normalizar a PA dos casos alterados, inclusive proporcionando melhora do condicionamento físico dos mesmos. Outra razão a ser analisada é se a ansiedade, o perfil sedentário e as práticas alimentares dos adolescentes causaram interferência para que não se obtivesse efeito positivo na PA.

Dessa forma, esses fatores não avaliados e que podem representar uma limitação desse estudo devem ser analisados em próximas pesquisas para se averiguar a real causa que propiciou a ausência de impacto do VGA no IMC e na PA dos adolescentes.

\section{DECLARAÇÃO DE CONFLITO DE INTERESSE:}

O presente artigo foi fruto de um trabalho de conclusão de curso apresentado ao curso de Bacharelado em Enfermagem da Universidade Estadual da ParaíbaUEPB.

\section{FONTE FINANCIADORA DO PROJETO:}

Este trabalho recebeu financiamento da Universidade Estadual da ParaíbaPROPESQ 2017. [Processo:4.06.01.00-5-415/2017-1]. 


\section{REFERÊNCIAS BIBLIOGRÁFICAS}

ABESO. Associação Brasileira para o Estudo da Obesidade e da Síndrome Metabólica. Diretrizes brasileiras de obesidade. São Paulo, 4.ed, 2016, p.186.

BIDDISS, E.; IRWIN, J. Active Video Games to Promote Physical Activity in Children and Youth. Arch Periatr Adolesc Med, Estados Unidos, v.164, n.7, p.664-672, 2010.

BIRO, F. M; WIEN, M. Childhood obesity and adult morbidities. American Society for Clinical Nutrition, Estados Unidos, v.91, n.5, p.1499S-505S, 2010.

$\mathrm{BLOCH}, \mathrm{K} . \mathrm{V}$ et al. ERICA: prevalências de hipertensão arterial e obesidade em adolescentes brasileiros. Rev Saúde Pública, v. 50, n. 1, p. 9s, 2016.

CABRERA, T. F. C et al. Análise da prevalência de sobrepeso e obesidade e do nível de atividade física em crianças e adolescentes de uma cidade do sudoeste de São Paulo. Revista Brasileira de Crescimento e Desenvolvimento Humano, São Paulo, v.24, n.1, p. 67-72, 2014.

CRAIGIE, A. M et al. Tracking of obesity-related behaviours from childhood to adulthood: a systematic review. Maturitas, v.70, n. 3, p. 266-284, 2011.

FRIEDEMANN, $\mathrm{C}$ et al. Cardiovascular disease risk in healthy children and its association with body mass index: systematic review and meta-analysis. BMJ, Grã-Bretanha, v. 345, p. e4759, 2012.

GAO, Z; CHEN, S; STODDEN, D.F. Comparação dos níveis de atividade física das crianças na educação física, recesso e exergaming. J Phys Act Health, Champaign, v.12, n.3, p: 349- 354, 2015.

MAILLOT, P; PERROT, A; HARTLEY, A. Effects of interactive physical-activity video- game training on physical and cognitive function in older adults. Psychol Aging, v.27, n.3, p:589-600, 2012.

MARQUES, J. P et al. A hipertensão arterial e o exercício físico: elementos para uma prescrição médica. Revista Portuguesa de Medicina Geral e Familiar, v. 31, n. 1, p. 46-50, 2015.

MELO, R. J. P et al. Respostas agudas da frequência cardíaca e da pressão arterial em uma sessão de jogos de vídeo game ativos em adultos saudáveis: um estudo piloto. Revista de Terapia Ocupacional da Universidade de São Paulo, São Paulo, v.24, n.3, p.259-266, 2014.

MORAES, L. I et al. Pressão Arterial Elevada em Crianças e sua Correlação com Três Definições de Obesidade Infantil. Arq Bras Cardiol, v.102, n.2, p.175-180, 2014.

NILSSON, $S$ et al. Active and passive distraction in children undergoing wound dressings. Journal of Pediatric Nursing, v.28, n.2, p.158-166, 2013.

PEREIRA, J. C et al. Exergames como alternativa para o aumento do dispêndio energético: uma revisão sistemática. Revista Brasileira de Atividade Física e Saúde, Pelotas, v.17, n.5, p.327-35, 2012.

PERRIER-MELO, R. J et al. Efeito do treinamento com videogames ativos nas dimensões morfológica e funcional: estudo clínico randomizado. Motricidade, vol.12, n.2, p.70-79, 2016.

QUEIROGA, M. R. et al. Caracterização do ambiente físico e prática de atividades físicas em 
unidades básicas de saúde de Guarapuava, Paraná, 2011-2012. Revista de Epidemiologia e Serviços de Saúde, v.25, n.4, p.827-836, 2016.

RAMOS, T. D. A et al. Assessment of the carotid artery intima-media complex through ultrasonography and the relationship with Pathobiological Determinants of Atherosclerosis in Youth. Cardiology in the Young, Reino Unido, v.26, n.7, p.1333-1342, 2015.

RAUBER, S. B et al. Variáveis cardiovasculares durante e após a prática do vídeo game ativo "Dance Dance Revolution" e televisão. Motriz, São Paulo, v.19, n.2, p.358-367, 2013.

RAUBER, S.B et al. Traditional games resulted in post-exercise hypotension and a lower cardiovascular response to the cold pressor test in healthy children. Front Physiol, v. 5, p.245, 2014.

ROWLAND, J. L et al. Perspectives on active video gaming as a new frontier in accessible physical activity for youth with physical disabilities. Physical therapy, Washington, v.96, n.4, p.521-532, 2016.

SBC. Sociedade Brasileira de Cardiologia. IV Diretriz Brasileira sobre Dislipidemias e Prevenção da Aterosclerose. Arq Bras Cardiol, v.88, p.18, 2007.

SELL, K; LILLIE, T; TAYLOR, J. Energy expenditure during physically interactive video game playing in male college students with different playing experience. Journal of American College Health, Estados Unidos, v. 56, n.5, p. 505-512, 2008.

SILVA, S. L et al. Influência de fatores antropométricos e atividade física na pressão arterial de adolescentes de Taguatinga, Distrito Federal, Brasil. Motricidade, v. 9, n. 1, 2013.

STAIANO, A.E; ABRAHAM, A.A; CALVERT, S.L. Adolescent exergame play for weight loss and psychosocial improvement: a controlled physical activity intervention. Obesity (Silver Spring), Mar, v.21, n.3, p:598-601, 2013.

TENÓRIO, M. C. M et al. Atividade física e comportamento sedentário em adolescentes estudantes do ensino médio. Rev Bras Epidemiol, v.13, n.1, p.105-17, 2010.

WHO. World Health Organization. Coming of age: adolescent health. Set. 2018. Disponível em: https://www.who.int/health-topics/adolescents/coming-of-age-adolescent-health. Acesso em: 08 jan. 2020.

WHO. World Health Organization. Obesity and overweight. Fev. 2018. Disponível em: https://www.who.int/news-room/fact-sheets/detail/obesity-and-overweight. Acesso em: 08 jan. 2020. 